\title{
Incidental Accumulation of Fluciclovine in Neuroendocrine Tumour in a Patient with Oncological Duplicity
}

\author{
Zuzana Balážováa, b Igor Černýa, b Petr Vyškovskýc \\ aDepartment of Radiology and Nuclear Medicine, University Hospital Brno, Brno, Czech \\ Republic; b Faculty of Medicine, Masaryk University, Brno, Czech Republic; 'Department of \\ Nuclear Medicine and PET/CT, Tomáš Bat’a Hospital Zlín, Zlín, Czech Republic
}

\section{Keywords}

Fluciclovine $\cdot$ Incidental uptake $\cdot$ Neuroendocrine tumour

\begin{abstract}
$18 \mathrm{~F}$-fluciclovine is a PET radiopharmaceutical used for the detection of recurrent prostate cancer in adult men after primary curative treatment with suspicion of recurrence based on elevated prostate-specific antigen level. Several incidental uptakes of $18 \mathrm{~F}$-fluciclovine in other tumour types have been described in the literature so far - in breast cancer, hepatocellular carcinoma, and malignant melanoma. Our case report presents a patient with oncological duplicity (prostate gland carcinoma and newly diagnosed neuroendocrine tumour) and with accumulation of fluciclovine in pathologically proved neuroendocrine tumour, later imagined also by octreotide SPECT/CT. To our knowledge, this is the first case of fluciclovine accumulated in a neuroendocrine tumour described in the literature.

\section{Introduction}

18F-fluciclovine (FCV) is a positron emission tomography (PET) radiopharmaceutical used for the detection of recurrent prostate cancer in adult men after primary curative treatment with the suspicion of recurrence based on elevated prostate-specific antigen (PSA) level [1]. The sensitivity of 18F-FCV PET for detecting recurrent disease increases with rising levels of PSA [2], the specificity for prostate bed in recurrent disease being $40-75 \%$, as the residual prostate gland tissue is prone to uptake due to inflammation or prostatic hyper- 

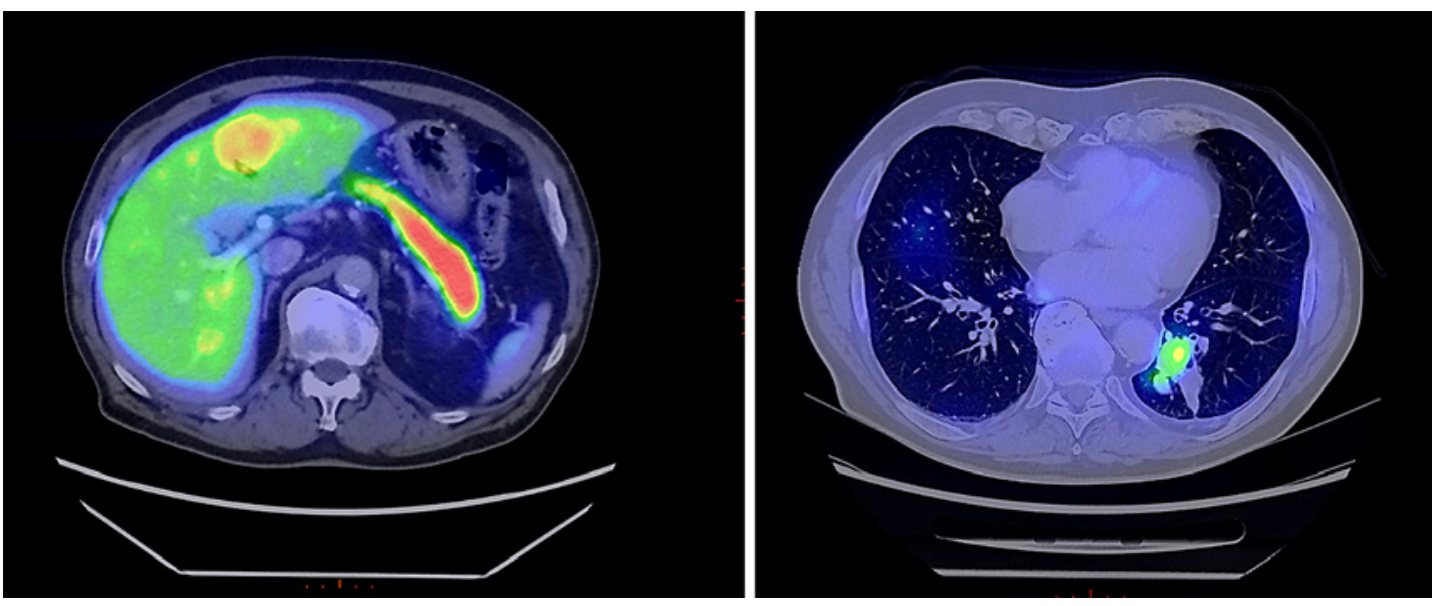

Fig. 1. Fluciclovine (FCV) PET/CT showing a tumorous mass with accumulating FCV in the liver (left) and in the lungs (right).

trophy, the specificity for extraprostatic lesions was reported 97 and $100 \%[1,3,4]$. FCV is accumulated by prostate cancer cells via specialised amino acids transporters, predominantly alanine-serine-cysteine transporter 2 (ASCT2) with contribution of l-amino acid transporter 1 (LAT-1) [1].

The fact that 18F-FCV can be accumulated by other cancer types is stated in the radiopharmaceutical information, however, these are not specified. Several incidental uptakes of 18F-FCV have been described -in male breast cancer, hepatocellular carcinoma, and malignant melanoma [5-7]. Accumulation of 18F-FCV in lesions other than cancer was described in superior sagittal sinus due to its dilatation [8].

\section{Case Presentation}

We present a case of a 78-year-old patient with a history of prostate cancer (February 2013: acinar adenocarcinoma, Gleason Score 3+3, T1cN0M0, PSA 7.91), who was treated by curative radiotherapy (June-September 2013, 78 Gy/39 fractions) with the following restaging examinations without any signs of relapse, the PSA trend: 1.14 (2014), 0.42 (2015), 0.47 (2016), 0.5 (2017), 0.3 (2018).

In 2018, the patient was admitted to our hospital for haemoptysis. The chest X-ray did not show any pathology; however, videofibrobronchoscopy proved a tumour in bronchopulmonary segment 10 on the left. The histological examination proved parts of bronchial mucosa with fragments of adenocarcinoma, with conclusion on a high suspicion of metastasis of acinar adenocarcinoma of the prostate gland. The CT scan proved a lung tumour in segment 10 on the left, several suspicious nodules in the left hilus, several liver metastases, and numerous osteoplastic bone metastases.

In October 2018, the patient underwent 18F-FCV PET with the following results: sclerotic metastatic lesions with higher accumulation of FCV in thoracic vertebrae 4 and 7, first sacral vertebra, and in the left ilium; tumorous lesion in the left lung obturating bronchi to segments 9 and 10 with higher cumulation of FCV (Fig. 1, left); three liver metastases, two of them with higher cumulation of FCV (Fig. 1, right). Focal accumulation of FCV in the prostate gland tumour; and no lymphadenopathy. 

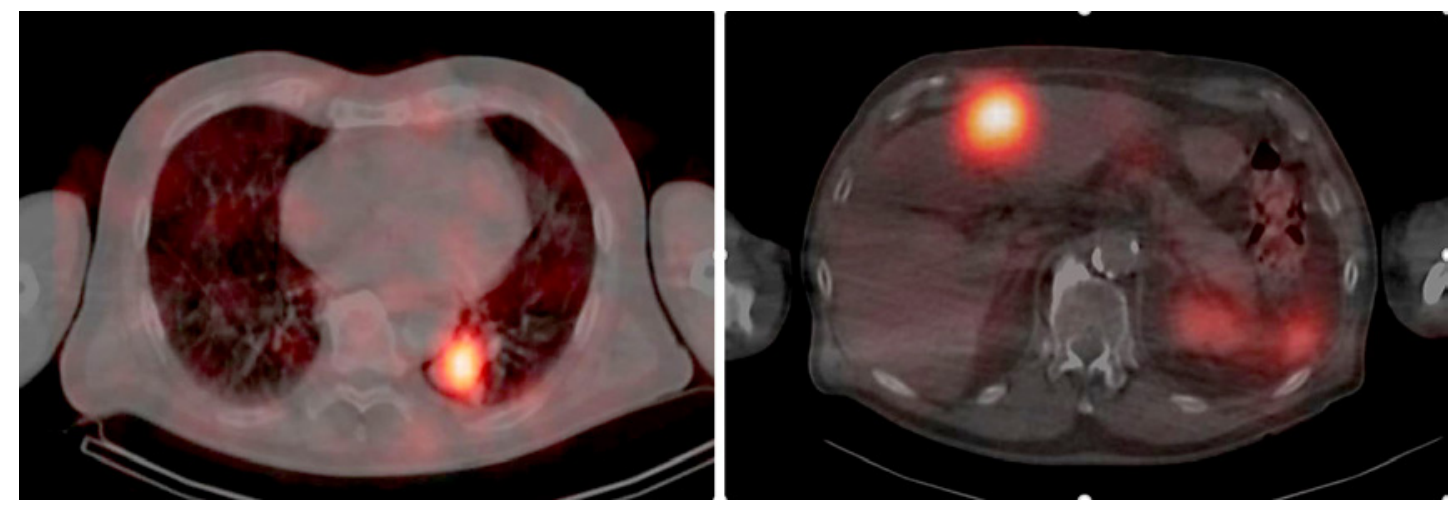

Fig. 2. Octreotide SPECT/CT showing the same tumorous masses as in Fig. 1, accumulating octreotide - in the lungs (left) and in the liver (right).

However, due to low levels of PSA (0.22 in October 2018), a second reading of histology from the lung tumour was requested. This proved positivity for chromogranin, with the conclusion of possible grade I typical carcinoid; however, the examination was limited due to a lack of preserved tumorous tissue.

Subsequently, a biopsy from the liver metastasis was performed, proving the same type of tumour as in the lungs after the second reading of histology, that is, neuroendocrine tumour (NET) grade 1-2. The patient underwent octreotide SPECT/CT (Fig. 2) in December 2018 with the following results: pathological cumulation of radiopharmaceutical in all stages (after 4 and $24 \mathrm{~h}$ ) found in the area of the left pulmonary hilus, in all three liver metastases, in the bone metastases in the fourth and seventh thoracic vertebrae, the third rib on the right, and in the left ilium.

Significant laboratory results include a chromogranin A level of $109 \mu \mathrm{g} / \mathrm{L}$ in serum, a hydroxyindole acetic acid level of $84.4 \mu \mathrm{mol} / \mathrm{L}$ in urine, and a hydroxyindole acetic acid level of $211 \mu \mathrm{mol} / \mathrm{L}$ in $24-\mathrm{h}$ urine collection $(2,500 \mathrm{~mL})$.

The patient is now on therapy with somatostatin analogue.

\section{Discussion/Conclusion}

In their paper, Schuster et al. [9] gave an extensive overview of both physiological uptake of FCV as well as incidental uptake, including inflammations and other cancer types; NET, however, is not listed.

We believe that LAT- 1 is what interconnects FCV and NET in our patient. LAT-1 expression in healthy adults is detected in the gastrointestinal mucosa, pancreatic islet cells, testicular Sertoli cells, and others [10]. It is overexpressed in some NET, such as pheochromocytoma, medullary thyroid carcinoma, and NET of the lungs. Its overexpression was proven in many types of solid tumours [11-13]. Our patient was diagnosed with grade I NET; however, in a study by Kaira et al. [13], all 10 patients diagnosed with grade I NET of the lung did not express LAT-1. Moreover, in a case study presented by Bosserman et al. [14], the authors describe a distinct imaging characteristics, i.e., the uptake of FCV and 68Ga-DOTATATE, in a patient with a history of histologically proven prostate cancer and rectal well-defined NET.

On the other hand, a case presented by Chen et al. [15] describes a patient with low PSA levels and a FCV PET/CT superscan with bone metastases confirmed by biopsy later. 
In conclusion, incidental FCV uptake in cancer types other than prostate cancer has already been described, with our case report adding to this list. FCV PET is a tool to detect recurrent prostate cancer with rising PSA levels. In our case, the patient had low PSA levels and FCV uptake in metastases which were later proven to be NET.

\section{Acknowledgement}

We would like to thank Dr. Pavel Veselý, PhD, clinical oncologist, for his help with the clinical data of the patient.

\section{Statement of Ethics}

Written informed consent was obtained from the patient for the publication of this case report and accompanying images.

\section{Disclosure Statement}

The authors have no conflicts of interest to declare.

\section{Funding Sources}

The authors declare no funding.

\section{Author Contributions}

Zuzana Balážová wrote the manuscript, conducted review, and interpreted the finding. Igor Černý acquired and interpreted the imaging data and revised the manuscript. Petr Vyškovský acquired and interpreted the imaging data and revised the manuscript.

\section{References}

1 Parent EE, Schuster DM. Update on 18F-fluciclovine PET for prostate cancer imaging. J Nucl Med. 2018 May; 59(5):733-9.

2 Akin-Akintayo 00, Jani AB, Odewole O, Tade FI, Nieh PT, Master VA, et al. Change in salvage radiotherapy management based on guidance with FACBC (fluciclovine) PET/CT in postprostatectomy recurrent prostate cancer. Clin Nucl Med. 2017 Jan;42(1):e22-8.

3 Evans JD, Jethwa KR, Ost P, Williams S, Kwon ED, Lowe VJ, et al. Prostate cancer-specific PET radiotracers: a review on the clinical utility in recurrent disease. Pract Radiat Oncol. 2018 Jan;8(1):28-39.

4 Odewole OA, Tade FI, Nieh PT, Savir-Baruch B, Jani AB, Master VA, et al. Recurrent prostate cancer detection with anti-3-[(18)F]FACBC PET/CT: comparison with CT. Eur J Nucl Med Mol Imaging. 2016 Sep;43(10):177383.

5 Gill HS, Tade F, Greenwald DT, Yonover PM, Savir-Baruch B. Metastatic male breast cancer with increased uptake on 18F-fluciclovine PET/CT scan. Clin Nucl Med. 2018 Jan;43(1):23-4.

6 Sannananja B, Shah HU, Behnia F. 18F-Fluciclovine uptake by an incidentally detected hepatocellular carcinoma in a case of biochemically recurrent prostate cancer. Clin Nucl Med. 2018 Sep;43(9):695-6.

7 Teoh EJ, Tsakok MT, Bradley KM, Hyde K, Subesinghe M, Gleeson FV. Recurrent malignant melanoma detected on 18F-fluciclovine PET/CT imaging for prostate cancer. Clin Nucl Med. 2017 Oct;42(10):803-4. 
8 Oldan JD, Miller SM, Barnes A, Khandani AH. Trapping of 18F-fluciclovine (FACBC) in superior sagittal sinus. Clin Nucl Med. 2019 Jan;44(1):48-9.

9 Schuster DM, Nanni C, Fanti S, Oka S, Okudaira H, Inoue Y, et al. Anti-1-amino-3-18F-fluorocyclobutane-1-carboxylic acid: physiologic uptake patterns, incidental findings, and variants that may simulate disease. J Nucl Med. 2014 Dec 1;55(12):1986-92.

10 Hayashi K, Anzai N. Novel therapeutic approaches targeting L-type amino acid transporters for cancer treatment. World J Gastrointest Oncol. 2017;9(1):21, 9.

11 Barollo S, Bertazza L, Watutantrige-Fernando S, Censi S, Cavedon E, Galuppini F, et al. Overexpression of L-type amino acid transporter 1 (LAT1) and 2 (LAT2): novel markers of neuroendocrine tumors. Fotiadis D, editor. PLoS One. 2016 May 25;11(5):e0156044.

12 Häfliger P, Charles R-P. The L-type amino acid transporter LAT1-an emerging target in cancer. IJMS. 2019 May 16;20(10): 2428.

13 Kaira K, Oriuchi N, Imai H, Shimizu K, Yanagitani N, Sunaga N, et al. Expression of L-type amino acid transporter 1 (LAT1) in neuroendocrine tumors of the lung. Pathol Res Pract. 2008 Aug;204(8):553-61.

14 Bosserman AJ, Dai D, Lu Y. Distinct imaging characteristics of different metastases from primary prostate adenocarcinoma and rectal carcinoid tumor on 18F-fluciclovine and 68Ga-DOTATATE PET/CT. Clin Nucl Med. 2019 Jan;44(1):83-4.

15 Chen B, Macapinlac HA, Lu Y. Superscan 18F-fluciclovine PET/CT of PSA-negative prostate cancer bone metastases. Clin Nucl Med. 2019 Apr;44(4):337-8. 